Research Paper

\title{
A novel circulating hsa_circ_0081001 act as a potential biomarker for diagnosis and prognosis of osteosarcoma
}

\author{
Zhu Kun-Peng ${ }^{* 1,2}$, Zhang Chun-Lin ${ }^{* 1,2 \bowtie}$, Hu Jian-Ping ${ }^{* 1,2}$, Zhang Lei ${ }^{1,2}$ \\ 1. Department of Orthopedics, Shanghai Tenth People's Hospital, Tongji University, School of Medicine, Shanghai 200072, PR China \\ 2. Institute of Bone Tumor Affiliated to Tongji University School of Medicine, Shanghai 200072, PR China \\ * Zhu Kun-Peng, Zhang Chun-Lin and Hu Jian-Ping contributed equally to this study and share first authorship. They are all considered co-first authors. \\ $\square$ Corresponding author: Zhang Chun-Lin, MD, Department of Orthopedics, Shanghai Tenth People's Hospital, Tongji University, School of Medicine, 301, \\ Yan-chang Middle Road, Shanghai 200072, China. E-mail: shzhangchunlin123@163.com. Fax: +86 13761904091. \\ (c) Ivyspring International Publisher. This is an open access article distributed under the terms of the Creative Commons Attribution (CC BY-NC) license \\ (https:// creativecommons.org/licenses/by-nc/4.0/). See http://ivyspring.com/terms for full terms and conditions.
}

Received: 2018.05.28; Accepted: 2018.07.14; Published: 2018.09.01

\begin{abstract}
Chemo-resistance and lung metastasis have been the two obstacles in the osteosarcoma (OS) treatment, which is still lack of effective biomarkers for prediction, diagnosis and treatment. Circular RNA (circRNA) is a new type of endogenous noncoding RNA that could serve as ideal biomarkers in cancer because of its stable loop structure. However, little is known about the diagnostic value of circRNAs in OS as well as their associations with clinicopathologic characteristics of OS patients. In the current study, we identified a novel circRNA, hsa_circ_0081001, screened by the RNA sequencing in the three paired chemo-resistant and chemo-sensitive OS cell lines (MG63/DXR vs MG63, KHOS/DXR vs KHOS, U2OS/DXR vs U2OS), and found that hsa_circ_0081001 was significantly up-regulated in the OS cell lines, tissues and serums, associated with poor overall survival and cox multivariate analysis showed that hsa_circ_0081001 was a novel independent prognostic factor for OS patients. Then, receiver operating characteristic (ROC) curve analysis revealed that hsa_circ_0081001 could act as a biomarker for the OS diagnosis and prognosis prediction, better than alkaline phosphatase (ALP) and lactate dehydrogenase (LDH). In addition, we preliminarily found that hsa_circ_0081001 expression level may dynamically monitor and reflect the condition changes of OS patients in a small-scale prospective clinical pretest. In conclusion, our study suggested that circulating hsa_circ_0081001 could serve as a potential biomarker and therapeutic target for OS patients.
\end{abstract}

Key words: osteosarcoma; circRNA; biomarker.

\section{Introduction}

Osteosarcoma (OS) is the most common primary malignant bone tumor in the children, adolescents and young adults [1]. Surgery with combinational chemotherapy has made limb salvage possible for most of patients and led to an increased 5 -year survival rate to about $70 \%-80 \%$ [2]. However, the patients who occurred in chemo-resistance and lung metastasis always had an unfavorable prognosis, with the markedly decreased 5-year survival rate to nearly 20\% [3]. Despite having unsatisfactory sensitivity and specificity, alkaline phosphatase (ALP) and lactate dehydrogenase (LDH) are still the most common serum markers used for diagnosing OS [4-7].
Therefore, it is very critical for OS patients to find some novel efficient biomarkers and targets for early diagnosis and treatment [8].

With the rapid development of high-throughput sequencing technology, more and more non-coding RNAs have been identified and paid more attention in the few decades [9, 10]. Of them, circular RNAs (circRNAs) are a large class of non-coding RNAs characterized by a covalently closed continuous loop with neither 5'-3' polarities nor polyadenylated tails. The closed loop structure has made it more stable than the linear RNA in response to the RNA exonuclease degradation [11]. Besides, most of 
circRNAs are evolutionarily conserved and often expressed in tissue-type-specific and developmental stage-specific manners [12]. Increasing evidence has shown that circRNAs could play an essential role in various cellular pathological and physiological processes, including carcinogenesis [13] and circRNA could be a promising and technically suitable biomarker for the cancer diagnosis and treatment [14, 15]. Actually, many circRNAs, such as hsa_circ_ 0001649, hsa_circ_0000190, hsa_circ_0013958, have been reported to be biomarkers in the hepatocellular carcinoma [16], gastric cancer [17], lung adenocarcinoma [18], respectively. However, there are seldom circRNAs reported to be a biomarker in the osteosarcoma.

In the current study, we identified a novel circRNA, hsa_circ_0081001, screened by the RNA sequencing in the three paired chemo-resistant and chemo-sensitive osteosarcoma cell lines (MG63/DXR vs MG63, KHOS/DXR vs KHOS, U2OS/DXR vs U2OS), and found that hsa_circ_0081001 was significantly up-regulated in the OS cell lines, tissues and serums, related to poor prognosis of OS patients. Then, we found that hsa_circ_0081001 could be used a promising biomarker for the osteosarcoma diagnosis and prognosis prediction through the ROC cure analysis, better than ALP and LDH. In addition, we preliminarily found that hsa_circ_0081001 expression level may dynamically monitor and reflect the condition changes of OS patients in a small-scale prospective clinical pretest, which may provide a new diagnostic tool in OS.

\section{Materials and Methods}

\section{Cell culture}

Three human osteosarcoma cell lines (MG63,KHOS,U2OS) were purchased from American Type Culture Collection and cultured in DMEM supplemented with 10\% FBS (Gibco, Gran Island, NY, USA), $100 \mathrm{U} / \mathrm{mL}$ of penicillin and $100 \mathrm{mg} / \mathrm{mL}$ of streptomycin (Invitrogen) at $37^{\circ} \mathrm{C}$ in a humidified $\mathrm{CO}_{2}$ $(5 \%)$ atmosphere. The paired three doxorubicinresistant osteosarcoma cell lines (MG63/DXR, KHOS/DXR, U2OS/DXR) were kindly donated by Dr.Yoshio Oda, Dr. Gonos ES and Dr. Duan ZF, respectively, which have been described in our previous study[19, 20].

\section{Patients and specimens}

A total of 82 primary osteosarcoma patients who received the same chemotherapy regimen before surgery and underwent complete resection surgery at Shanghai Tenth People's Hospital between 2006 and 2017 were included in this study. The study was approved by the Ethics Committee of Shanghai Tenth
People's Hospital, and written informed consent was obtained from all the participants. All patients' slides were reviewed to confirm the diagnosis and to classify the tumor according to Enneking Stage. Besides, we collected blood samples $(5 \mathrm{ml}$, peripheral vein) from 50 OS patients before surgery, other 30 patients with benign bone tumor (ten cases of giant cell tumor of bone, eight cases of osteoclastoma, twelve cases of fibrous dysplasia) and 20 age- and sex-matched healthy volunteers as the control group. All the resected or biopsy specimens were placed immediately into liquid nitrogen and stored at $-80^{\circ} \mathrm{C}$. The serum extracted from the blood samples were collected using standard procedures. According to the Huvos scoring system [21], the patients were classified as chemo-resistant and chemo-sensitive groups. ALP and LDH data were simultaneously collected and analyzed through the routine liver function monitoring. The clinical parameters of osteosarcoma patients in this study are shown in Table 1.

Table 1. Clinical parameters of osteosarcoma patients enrolled in this study

\begin{tabular}{|c|c|c|c|c|}
\hline \multirow[t]{2}{*}{ Pathological characteristics } & \multirow[t]{2}{*}{ Cases (n) } & \multicolumn{2}{|c|}{ hsa_circ_0081001 expression } & \multirow[t]{2}{*}{ Pvalue } \\
\hline & & High(27) & Low(55) & \\
\hline \multicolumn{5}{|l|}{ Gender } \\
\hline Male & $49(59.8 \%)$ & $17(63 \%)$ & $32(58.2 \%)$ & NS \\
\hline Female & $33(40.2 \%)$ & $10(37 \%)$ & $23(41.8 \%)$ & \\
\hline \multicolumn{5}{|l|}{ Age } \\
\hline$\geq 25$ & $24(29.3 \%)$ & $10(37 \%)$ & $14(25.5 \%)$ & NS \\
\hline$<25$ & $58(70.7 \%)$ & $17(63 \%)$ & $41(74.5 \%)$ & \\
\hline Location & & & & NS \\
\hline Distal of Femur & $38(46.3 \%)$ & $12(44.4 \%)$ & $26(47.3 \%)$ & \\
\hline Proximal of Tibia & $28(34.1 \%)$ & $10(37.0 \%)$ & $18(32.7 \%)$ & \\
\hline Other & $16(19.6 \%)$ & $5(18.6 \%)$ & $11(20 \%)$ & \\
\hline Enneking stage & & & & 0.046 \\
\hline I+IIA & $23(28 \%)$ & $3(11.1 \%)$ & $20(36.4 \%)$ & \\
\hline IIB/III & $59(72 \%)$ & $24(88.9 \%)$ & $35(63.6 \%)$ & \\
\hline Lung Metastasis & & & & 0.024 \\
\hline Yes & $25(30.5 \%)$ & $21(77.8 \%)$ & $4(7.3 \%)$ & \\
\hline No & $57(69.5 \%)$ & $6(22.2 \%)$ & $51(92.7 \%)$ & \\
\hline Chemoresistant & & & & 0.012 \\
\hline Yes & $32(39 \%)$ & $22(81.5 \%)$ & $10(18.2 \%)$ & \\
\hline No & $50(61 \%)$ & $5(18.5 \%)$ & $45(81.8 \%)$ & \\
\hline
\end{tabular}

\section{Next generation RNA sequencing analysis}

Total RNA from three paired chemo-resistant and chemo-sensitive osteosarcoma cell lines were treated with mirVana miRNA Isolation Kit (Ambion, Texas, USA) and RNAse R (Epicenter, CA, USA) to remove ribosomal and linear RNA. The quantity and quality of total RNA samples were measured using NanoDrop ND-1000 (Wilmington, DE, USA). RNA integrity was evaluated using the Agilent 2100 Bioanalyzer (Agilent Technologies, Santa Clara, CA, USA). The samples with RNA Integrity Number (RIN) $\geq 7$ were subjected to the subsequent analysis. The libraries were constructed using TruSeq Stranded Total RNA according to the manufacturer's 
instructions. Then these libraries were sequenced on the Illumina sequencing platform (HiSeqTM 2500) and $150 \mathrm{bp} / 125 \mathrm{bp}$ paired-end reads were generated. All the sequencing procedures and analyses were performed in OEbiotech (Shanghai, China).

\section{RNA isolation and quantitative real-time PCR (qRT-PCR)}

Total RNA was isolated from cells, tissues or serums using the TRIzol kit (Invitrogen, Carlsbad, CA, USA) following to the manufacture's guide. Quantitative real-time PCR (qRT-PCR) analysis was performed to detect the hsa_circ_0081001 expression using SYBR green kit (TaKaRa, Dalian, China) on the Light Cycler 480 (Roche, Switzerland) in accordance with the instructions. The expression of hsa_circ_0081001 was normalized to GAPDH. Divergent primers were shown as follows: hsa_circ_0081001 forward primers: 5'-CATGCAGCCT GGCTCTTACC-3', reverse primers: 5'-CTGCTCCAA GAAAACCTGAAACT-3'; GAPDH forward primers: 5'-AATGGGCAGCCGTTAGGAAA-3', reverse primers: 5'-TGAAGGGGTCATTGATGGCA-3'.

\section{Statistical analysis}

All statistical analyses were performed using SPSS 22.0 software (IBM) and Graphpad. Differences between OS tissues and paired adjacent nontumorous tissues were analyzed using paired $t$ test. The correlations between circRNA expression levels and clinicopathological factors were further analyzed by one-way analysis of variance (ANOVA). Overall survival were calculated by Kaplan-Meier survival analysis and compared using the log-rank test. The joint effect of covariables was examined using the Cox proportional hazards regression model. A receiver operating characteristic (ROC) curve was established to determine the efficiency as a biomarker. A combined ROC was calculated based on the logistic regression model. Differences were considered statistically significant when $P$ values $<0.05$.

\section{Results}

\section{Hsa_circ_0081001 was significantly up-regulated in the OS cell lines and tissues, correlated with poor clinical outcomes}

To find the specific circRNA in the osteosarcoma, we first screened the circRNA expression profile in the three paired chemo-resistant and chemo-sensitive osteosarcoma cell lines (MG63/DXR vs MG63, $\mathrm{KH}-\mathrm{OS} / \mathrm{DXR}$ vs KH-OS, U2-OS/DXR vs U2-OS) and found 80 circRNAs were dysregulated, with 57 up-regulation and 23 down-regulation. Of them, hsa_circ_0081001 was up-regulated with 12 fold change in the chemo-resistant OS cell lines compared to the controlled (Fig. 1A). qRT-PCR results showed the consistency with RNA sequencing (Fig. 1B). Then we examined the expression of hsa_circ_0081001 in the 82 OS tissues and paired adjacent non-tumor tissues and found that hsa_circ_0081001 was markedly overexpressed in the OS tissues relative to the controlled (Fig. 1C). Besides, we divided the 82 patients into different groups, including I+IIA or IIB+III grade groups, chemo-resistant or chemo-sensitive groups and lung metastasis or non-lung metastasis groups, according to the medical records. As was illustrated in the Figure, hsa_circ_0081001 expression was higher in the IIB+III group, chemo-resistant group and lung metastasis group compared to the controlled groups $(\mathrm{P}<0.01$, Figure 1D-F).

In addition, we further divided the 82 patients into two groups with high or low hsa_circ_0081001 expression based on the average expression level and further K-M survival analysis demonstrated that patients with high hsa_circ_0081001 expression have shorter overall survival lifetime than those with low hsa_circ_0081001 expression.

Furthermore, we used cox proportional hazards regression model to evaluate whether the hsa_circ_0081001 expression could be used to predict the overall survival of OS patients. The results of univariate and multivariate survival analyses revealed that hsa_circ_0081001 as well as chemo-resistance, lung metastasis and Enneking stage, other than age, gender, tumor size or site could be considered as an independent prognostic factor for OS (Table 2). These results indicated the potential oncogenic role of hsa_circ_0081001 in OS.

Table 2. Univariate and multivariate Cox regression analyses of the relationship between hsa_circ_0081001 level, clinicopathological characteristics and survival of patients with osteosarcoma

\begin{tabular}{|c|c|c|c|c|c|}
\hline \multirow[t]{2}{*}{ Parameters } & \multirow[t]{2}{*}{ Categories } & \multicolumn{2}{|c|}{$\begin{array}{l}\text { Univariate } \\
\text { analysis }\end{array}$} & \multicolumn{2}{|c|}{$\begin{array}{l}\text { Multivariate } \\
\text { analysis }\end{array}$} \\
\hline & & HR & Pvalue & HR & Pvalue \\
\hline Age(years) & $\leq 25,>25$ & 0.642 & NS & 0.763 & NS \\
\hline Sex & Male, female & 0.836 & NS & 0.592 & NS \\
\hline Tumor location & $\begin{array}{l}\text { Femur and tibia, } \\
\text { others }\end{array}$ & 0.722 & NS & 0.681 & NS \\
\hline Enneking stage & I+IIA, IIB+III & 2.214 & 0.025 & 2.015 & 0.032 \\
\hline Lung Metastasis & Negative, positive & 3.232 & $<0.01$ & 3.014 & $<0.01$ \\
\hline Chemo-resistance & Negative, positive & 4.625 & $<0.001$ & 4.102 & $<0.001$ \\
\hline $\begin{array}{l}\text { hsa_circ_0081001 } \\
\text { expression }\end{array}$ & Low, high & 3.81 & $<0.01$ & 3.122 & $<0.01$ \\
\hline
\end{tabular}

Serum hsa_circ_0081001 may be a better diagnostic biomarker than ALP and LDH in OS

We then examined the expression level of hsa_circ_0081001 in serum samples from 50 OS patients, 30 benign bone tumor patients and 20 age- 

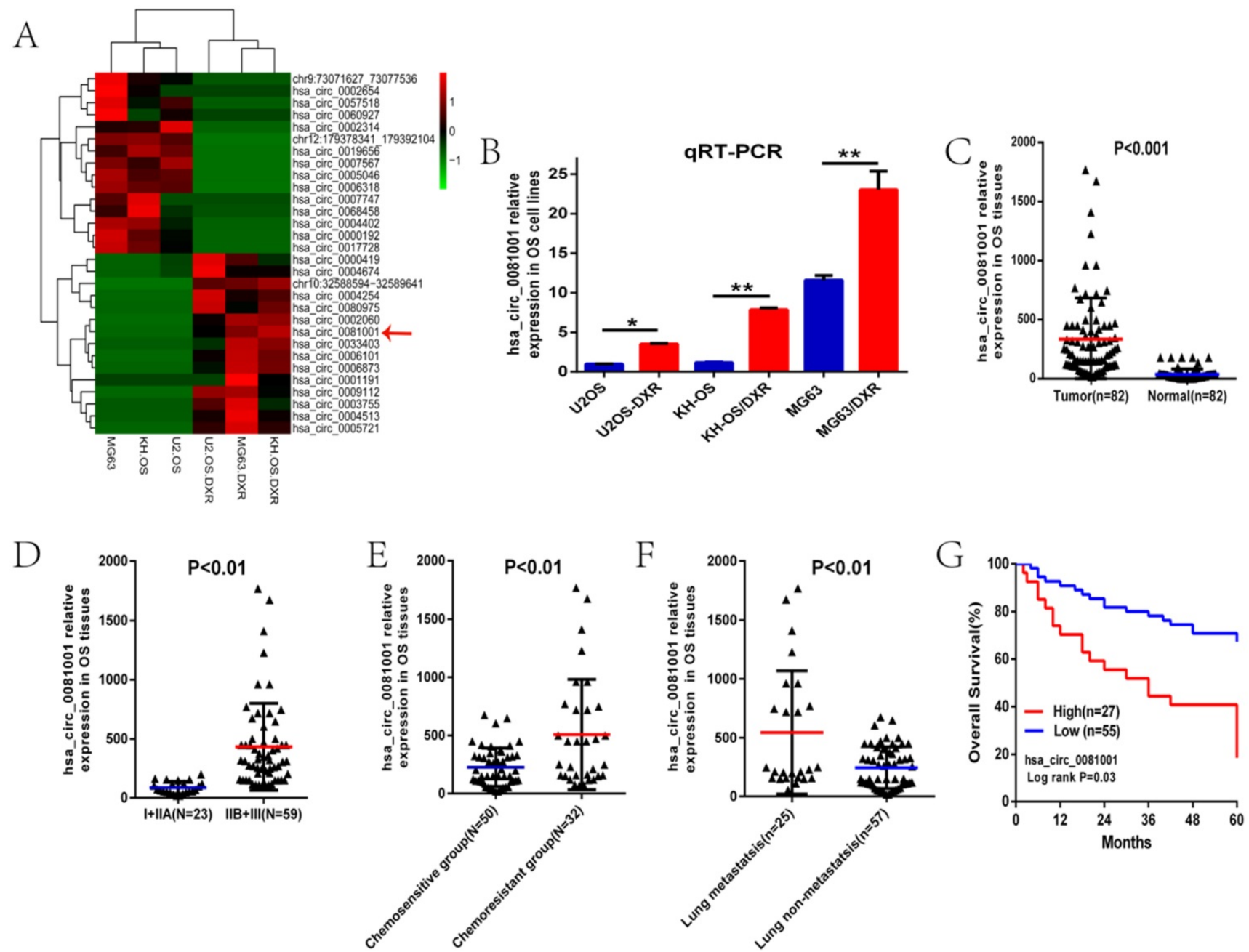

Figure 1. Hsa_circ_0081001 was significantly up-regulated in the OS cell lines and tissues, correlated with poor clinical outcomes. (A) Top 15 up-regulated and down-regulated differently expressed circRNAs screened by RNA sequencing in the three paired chemo-resistant and chemo-sensitive osteosarcoma cell lines. Of them, hsa circ 0081001 was up-regulated with 12 fold change in the chemo-resistant OS cells compared with the controlled. (B) Expression level of hsa circ 0081001 in the three paired chemo-resistant and chemo-sensitive OS cell lines by qRT-PCR. (C) Expression level of hsa_circ_0081001 in 82 paired OS and paracancerous tissues. (D) Expression level of hsa_circ_0081001 in OS tissues of patients in I+IIA and IIB+III groups. (E) Expression level of hsa_circ_0081001 in OS tissues of chemo-resistant and chemo-sensitive groups. (F) Expression level of hsa_circ_0081001 in OS tissues of lung metastasis and lung non-metastasis groups. (G) Patients with high hsa_circ_0081001 expression had a decreased overall survival time than those with low level of hsa_circ_0081001 expression.

and sex-matched healthy individuals as controlled to explore its diagnostic value as a potential biomarker for OS. As was shown in the Figure, hsa_circ_0081001 expression was gradually increased in the benign bone tumor and OS compared with the controlled (Fig. 2A). Besides, we found that hsa_circ_0081001 expression level markedly decreased after preoperative chemotherapy of two cycles and after operation (Fig. 2B-C). According to the grouping method previously described, we further found that serum hsa_circ_0081001 expression was higher in the chemo-resistant, lung metastasis or recurrence groups than the controlled, consistent with the results in the tissues (Fig. 2D-F).

To investigate the diagnostic value of hsa_circ_0081001 in distinguishing patients from OS and the controlled healthy individuals, ROC curve was used and the results showed that the area under the ROC curve (AUC) was 0.898. In addition, we simultaneously compared the effectiveness of hsa_circ_0081001, ALP, and LDH, which are two biomarkers commonly used in clinical as diagnostic biomarker in OS. Obviously, as was shown in the Fig 2G-H, hsa_circ_0081001 was more reliable to separate osteosarcoma from healthy individuals (AUC $=0.898$, $\mathrm{P}<0.001)$ than ALP $(\mathrm{AUC}=0.673, \mathrm{P}=0.026)$ and $\mathrm{LDH}$ (AUC $=0.80, \quad P<0.05$ ). Besides, we compared the diagnosed value of hsa_circ_0081001 with a circRNA biomarker of OS, circPVT1, previously reported by us in the same training set [22] and the result showed that AUC value of hsa_circ_0081001 is comparable to circPVT1(AUC 0.898 vs $0.871, \mathrm{P}>0.05$ ). In our study, circRNA (hsa_circ_0081001 and circPVT1) and tumor markers (ALP and LDH) were also combined into a single diagnostic panel after a logistic regression analysis in the training set. The integration analysis 
showed that hsa_circ_0081001 as well as hsa_circ_0081001 combined with ALP has the most AUC value, better than ALP, LDH, circPVT1 or other combinations (data not shown). The above results demonstrated the possible clinical significance of hsa_circ_0081001 as a diagnostic biomarker in OS.
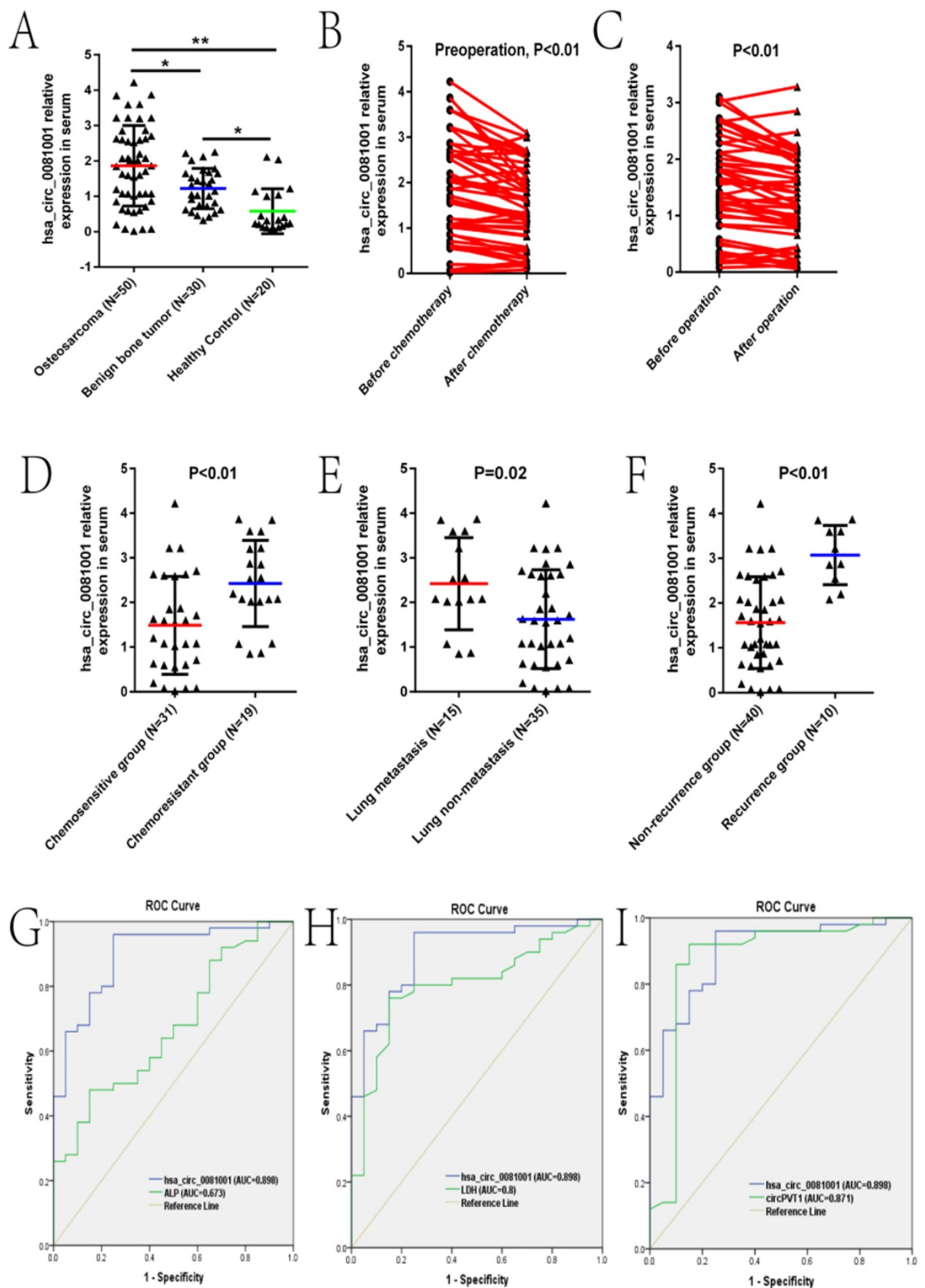

Figure 2. Serum hsa circ 0081001 may be a better diagnostic biomarker than ALP and LDH in OS. (A) Expression level of hsa circ 0081001 in serum from 50 OS patients, 30 benign bone tumor patients and 20 age- and sex-matched healthy volunteers. (B) Expression level of hsa_circ_0081001 in serum of 50 patients before and after preoperative chemotherapy. (C) Expression level of hsa_circ_0081001 in serum of 50 patients before and after operation. (D) Expression level of hsa_circ_0081001 in serum of chemo-resistant and chemo-sensitive groups. (E) Expression level of hsa circ 0081001 in serum of lung metastasis and lung non-metastasis groups. ( $F$ ) Expression level of hsa_circ_0081001 in serum of recurrence and non-recurrence groups. (G-I) ROC curves of the serum hsa_circ_0081001, ALP, LDH and circPVT1 in 50 newly diagnosed patients and 20 healthy donors. 


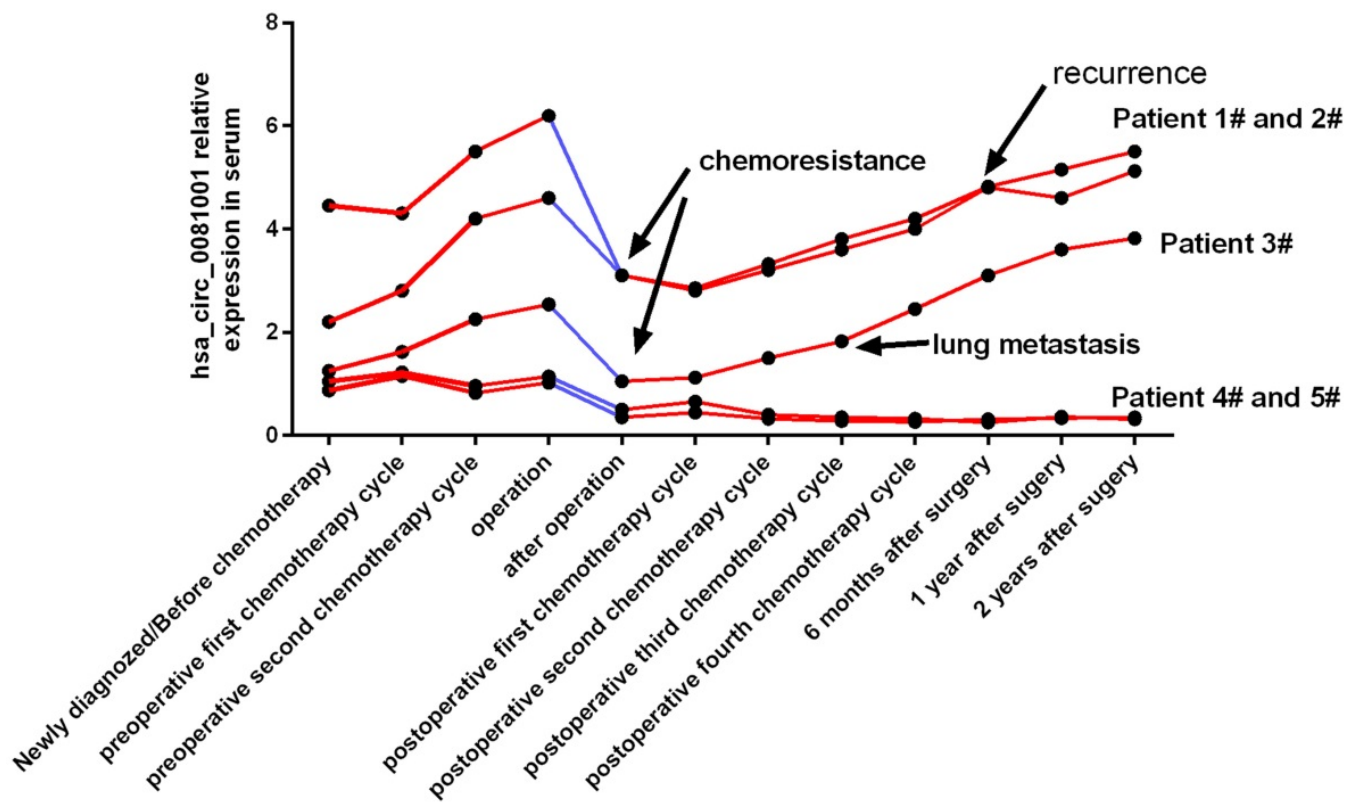

Figure 3. Serum hsa_circ_0081001 could dynamically monitor the condition changes of OS patients in a prospective clinical pretest.

\section{Serum hsa_circ_0081001 could dynamically monitor the condition changes of OS patients}

Owing to the lower incidence rate and the novel circRNA, to further demonstrate the clinical significance and potential application of hsa_circ_0081001, we tried to test its feasibility and reliability in a small-scale prospective clinical pretest. We then enrolled another five newly diagnosed OS patients from 2016, Jan and 2017, Jan, collected their serum samples in every treatment stage, and analyzed the correlation of hsa_circ_0081001 expression level with condition changes of OS patients. As was shown in the figure, in patient $1 \#, 2 \#$ and $3 \#$, hsa_circ_0081001 level in serum gradually increased in the preoperative chemotherapy, greatly decreased after surgery, and increased again in the postoperative chemotherapy. All of them were verified as chemo-resistance according to the evaluation of tumor necrosis rate in the resected tumor tissues. Patient 1\# and 2\# eventually suffered tumor recurrence 6 months after surgery and patient $3 \#$ suffered lung metastasis in the postoperative third chemotherapy cycle. However, in patient 4\# and 5\#, both of them are chemo-sensitive, occur surgery, and have a good prognosis without recurrence or lung metastasis during the 2-year follow-up period. Hsa_circ_0081001 level in their serum was initially lower than the other 3 patients, not greatly increased in the period of chemotherapy, and always maintained in a low level after operation. Therefore, the above results indicated that serum hsa_circ_0081001 level may early and accurately reflect the condition changes of OS patients, especially for the chemo-response and lung metastasis, with a potential value of clinical application in predicting OS progression.

\section{Discussion}

Chemo-resistance and lung metastasis have been the two obstacles in the OS treatment [23]. Until now, there is lack of effective biomarker to early diagnose OS and predict the OS progression, especially for the chemo-resistance and lung metastasis [24]. Uncovering the novel biomarker is very important for the OS diagnosis and treatment.

Owing to the stable characteristic of loop structure, many circRNAs have been reported to be biomarkers in various cancers $[25,26]$. For example, Zhang S et al [27] reported hsa_circ_0014130 as a new circular RNA biomarker in non-small cell lung cancer. Xie Y et al [28] found that hsa_circ_0074362 probably plays a role in the initiation of gastric cancer and may be a potential biomarker of gastric cancer. Zhang $X$ et al [29] showed that hsa_circ_0001649 could act as a novel prognostic biomarker and a potential therapeutic target for hepatocellular carcinoma patients. Besides, Yang F et al [30] found that circular RNA circ-LDLRAD3 could be a biomarker in diagnosis of pancreatic cancer. Zhuo $F$ et al [31] reported that circRNA0003906 serves as a potential biomarker for the diagnosis and treatment of colorectal cancer. $\mathrm{Li} \mathrm{W}$ et al [32] showed hsa_circ_0004277 as a new biomarker for acute myeloid leukemia. However, there have been seldom reports about circRNA as biomarker in OS progression.

In the present study, we first screened the circRNA expression profile in three paired 
chemo-resistant and chemo-sensitive OS cell lines by RNA sequencing and found that a novel circRNA, hsa_circ_0081001, was up-regulated in the chemo-resistant OS cell lines compared with the controlled, which was also verified by qRT-PCR. Then, we further found that hsa_circ_0081001 expression was significantly up-regulated in the OS tissues relative to the paratumor tissues. Besides, we found that hsa_circ_0081001 expression was greatly increased in the IIB+III group, chemo-resistant group and lung metastasis group compared to the controlled groups, related to poor prognosis of OS patients. Further multivariate survival analysis revealed that hsa_circ_0081001 as well as chemo-resistance, lung metastasis and Enneking stage could act as an independent prognostic factor for OS.

Then, we further detected the hsa_circ_0081001 expression level in serum from patients with OS or benign bone tumor and the controlled healthy individuals to explore its possibility to be a biomarker of OS. The results indicated that hsa_circ_0081001 expression was gradually increased in the benign bone tumor and OS compared with the controlled. Then we further found that serum hsa_circ_0081001 expression level markedly decreased after preoperative chemotherapy of two cycles and after operation. And hsa_circ_0081001 expression was higher in the chemo-resistant, lung metastasis or recurrence groups than the controlled, consistent with the results in the tissues. Besides, ROC curve analysis further demonstrated that hsa_circ_0081001 may be a better diagnostic biomarker than ALP and LDH, two biomarkers commonly used in clinical [33], with more sensitivity and specificity.

Actually, a previous study by Song $\mathrm{YZ}$ et al found that hsa_circ_0001564 was significantly overexpressed in OS tissue, as well as in OS cell lines and could act as a potential biomarker for the OS [34]. But they did not analyze the correlation of hsa_circ_0001564 expression level with OS progression, such as chemo-resistance, lung metastasis, and overall survival time and did not examine the hsa_circ_0001564 expression in serum of OS patients. Besides, in a previous study by us, we first reported that circPVT1, a potential new circular RNA biomarker, contributes to doxorubicin and cisplatin resistance of OS cells by regulating ABCB1 [22]. To further demonstrate the feasibility of hsa_circ_0081001, we compared its specificity and sensitivity with circPVT1 by ROC curve and found that AUC value of hsa_circ_0081001 is comparable to circPVT1 (AUC 0.898 vs 0.871, P>0.05), with an important value as a biomarker in OS.

In addition, we performed a small-scale prospective clinical pretest to demonstrate whether the changes of hsa_circ_0081001 expression level could dynamically monitor and reflect the condition changes of OS patients. Preliminary results indicated that the changes of hsa_circ_0081001 expression could predict the pivotal condition changes of OS patients, especially for chemo-resistance and lung metastasis. Obviously, a large-scale prospective multi-center clinical trial with more enrolled patients and more careful experimental design should be conducted to further identify the value of hsa_circ_0081001 in predicting OS progression. Besides, the function and regulatory mechanism of hsa_circ_0081001 in OS progression in vitro and in vivo should be clarified in the further study.

In summary, the current study identified that a novel circRNA, hsa_circ_0081001, was up-regulated in OS cell lines, tissues and serums, correlated with poor prognosis and may be a better diagnostic biomarker than ALP and LDH in OS. In addition, preliminary results indicated that hsa_circ_0081001 expression level may dynamically monitor and reflect the condition changes of OS patients. Our findings first shed light on the role of hsa_circ_0081001 as a novel circulating biomarker for the diagnosis and treatment target of OS.

\section{Abbreviations}

OS: Osteosarcoma; ALP: Alkaline phosphatase; LDH: Lactate dehydrogenase; circRNA: circular RNA; qRT-PCR: quantitative real-time polymerase chain reaction; ROC: Receiver operating characteristic; AUC: Area under the ROC curve.

\section{Acknowledgments}

This project was supported by a Grant from the National Natural Science Foundation of China (No.81572630), the Fundamental Research Funds for the Central Universities (NO.22120170216), Shanghai Pujiang Program of Shanghai Science and Technology Commission (NO.13PJD023) and Shanghai Jiaotong University Medical-Engineering Cross Research Fund (NO.YG2012MS49).

\section{Competing Interests}

The authors have declared that no competing interest exists.

\section{References}

1. Saraf AJ, Fenger JM, Roberts RD. Osteosarcoma: Accelerating Progress Makes for a Hopeful Future. Frontiers in oncology. 2018; 8: 4.

2. Yang $Y$, Han L, He Z, Li X, Yang S, Yang J, et al. Advances in limb salvage treatment of osteosarcoma. Journal of bone oncology. 2018; 10: 36-40.

3. Verrecchia F, Redini F. Transforming Growth Factor-beta Signaling Plays a Pivotal Role in the Interplay Between Osteosarcoma Cells and Their Microenvironment. Frontiers in oncology. 2018; 8: 133.

4. Fu Y, Lan T, Cai H, Lu A, Yu W. Meta-analysis of serum lactate dehydrogenase and prognosis for osteosarcoma. Medicine. 2018; 97: e0741. 
5. Han J, Yong B, Luo C, Tan P, Peng T, Shen J. High serum alkaline phosphatase cooperating with MMP-9 predicts metastasis and poor prognosis in patients with primary osteosarcoma in Southern China. World journal of surgical oncology. 2012; 10: 37.

6. Shimose S, Kubo T, Fujimori J, Furuta T, Ochi M. A novel assessment method of serum alkaline phosphatase for the diagnosis of osteosarcoma in children and adolescents. Journal of orthopaedic science : official journal of the Japanese Orthopaedic Association. 2014; 19: 997-1003.

7. Marais LC, Bertie J, Rodseth R, Sartorius B, Ferreira N. Pre-treatment serum lactate dehydrogenase and alkaline phosphatase as predictors of metastases in extremity osteosarcoma. Journal of bone oncology. 2015; 4: 80-4.

8. Harrison DJ, Geller DS, Gill JD, Lewis VO, Gorlick R. Current and future therapeutic approaches for osteosarcoma. Expert review of anticancer therapy. 2018; 18: 39-50.

9. de Almeida RA, Fraczek MG, Parker S, Delneri D, O'Keefe RT. Non-coding RNAs and disease: the classical ncRNAs make a comeback. Biochemical Society transactions. 2016; 44: 1073-8.

10. Guan D, Zhang W, Zhang W, Liu GH, Belmonte JC. Switching cell fate, ncRNAs coming to play. Cell death \& disease. 2013; 4: e464.

11. Wilusz JE. A 360 degrees view of circular RNAs: From biogenesis to functions. Wiley interdisciplinary reviews RNA. 2018: e1478.

12. Lei K, Bai H, Wei Z, Xie C, Wang J, Li J, et al. The mechanism and function of circular RNAs in human diseases. Experimental cell research. 2018;368: 147-158.

13. Chen S, Zhao Y. Circular RNAs: Characteristics, function, and role in human cancer. Histology and histopathology. 2018: 11969.

14. Zhang M, Xin Y. Circular RNAs: a new frontier for cancer diagnosis and therapy. Journal of hematology \& oncology. 2018; 11: 21.

15. Bolha L, Ravnik-Glavac M. Circular RNAs: Biogenesis, Function, and a Role as Possible Cancer Biomarkers. 2017; 2017: 6218353.

16. Oin M, Liu G, Huo X, Tao X, Sun X, Ge Z, et al. Hsa circ 0001649: A circular RNA and potential novel biomarker for hepatocellular carcinoma. Cancer biomarkers : section A of Disease markers. 2016; 16: 161-9.

17. Chen S, Li T, Zhao O, Xiao B, Guo J. Using circular RNA hsa circ 0000190 as a new biomarker in the diagnosis of gastric cancer. Clinica chimica acta; international journal of clinical chemistry. 2017; 466: 167-71.

18. Zhu X, Wang X, Wei S, Chen Y, Chen Y, Fan X, et al. hsa_circ_0013958: a circular RNA and potential novel biomarker for lung adenocarcinoma. The FEBS journal. 2017; 284: 2170-82.

19. Kun-Peng $Z$, Xiao-Long $M$, Chun-Lin Z. LncRNA FENDRR sensitizes doxorubicin-resistance of osteosarcoma cells through down-regulating ABCB1 and ABCC1. Oncotarget. 2017; 8: 71881-93.

20. Zhang $\mathrm{CL}$, Zhu KP, Ma XL. Antisense IncRNA FOXC2-AS1 promotes doxorubicin resistance in osteosarcoma by increasing the expression of FOXC2. Cancer letters. 2017; 396: 66-75.

21. Xu M, Jin H, Xu CX, Bi WZ, Wang Y. MiR-34c inhibits osteosarcoma metastasis and chemoresistance. Medical oncology (Northwood, London, England). 2014; 31: 972

22. Kun-Peng Z, Xiao-Long M, Chun-Lin Z. Overexpressed circPVT1, a potential new circular RNA biomarker, contributes to doxorubicin and cisplatin resistance of osteosarcoma cells by regulating ABCB1. International journal of biological sciences. 2018; 14: 321-30.

23. Luetke A, Meyers PA, Lewis I, Juergens H. Osteosarcoma treatment - where do we stand? A state of the art review. Cancer treatment reviews. 2014; 40: 523-32.

24. Zhou W, Hao M, Du X, Chen K, Wang G, Yang J. Advances in targeted therapy for osteosarcoma. Discovery medicine. 2014; 17: 301-7.

25. Han YN, Xia SQ, Zhang YY, Zheng JH, Li W. Circular RNAs: A novel type of biomarker and genetic tools in cancer. Oncotarget. 2017; 8: 64551-63.

26. Zhang Y, Liang W, Zhang P, Chen J, Qian H, Zhang X, et al. Circular RNAs: emerging cancer biomarkers and targets. Journal of experimental \& clinical cancer research : CR. 2017; 36: 152.

27. Zhang S, Zeng X, Ding T, Guo L, Li Y, Ou S, et al. Microarray profile of circular RNAs identifies hsa_circ_0014130 as a new circular RNA biomarker in non-small cell lung cancer. Scientific reports. 2018; 8: 2878 .

28. Xie Y, Shao Y, Sun W, Ye G, Zhang X, Xiao B, et al. Downregulated expression of hsa_circ_0074362 in gastric cancer and its potential diagnostic values. Biomarkers in medicine. 2018; 12: 11-20.

29. Zhang X, Qiu S, Luo P, Zhou H, Jing W, Liang C, et al. Down-regulation of hsa_circ_0001649 in hepatocellular carcinoma predicts a poor prognosis. Cancer biomarkers : section A of Disease markers. 2018; 22: 135-42.

30. Yang F, Liu DY, Guo JT, Ge N, Zhu P, Liu X, et al. Circular RNA circ-LDLRAD3 as a biomarker in diagnosis of pancreatic cancer. World journal of gastroenterology. 2017; 23: 8345-54.

31. Zhuo F, Lin $\mathrm{H}$, Chen $\mathrm{Z}$, Huang $\mathrm{Z}, \mathrm{Hu}$ J. The expression profile and clinical significance of circRNA0003906 in colorectal cancer. OncoTargets and therapy. 2017; 10: 5187-93.

32. Li W, Zhong C, Jiao J, Li P, Cui B, Ji C, et al. Characterization of hsa circ 0004277 as a New Biomarker for Acute Myeloid Leukemia via Circular RNA Profile and Bioinformatics Analysis. International journal of molecular sciences. 2017; 18. pii: E597.

33. Ren HY, Sun LL, Li HY, Ye ZM. Prognostic Significance of Serum Alkaline Phosphatase Level in Osteosarcoma: A Meta-Analysis of Published Data. BioMed research international. 2015; 2015: 160835.
34. Song YZ, Li JF. Circular RNA hsa_circ_0001564 regulates osteosarcoma proliferation and apoptosis by acting miRNA sponge. Biochemical and biophysical research communications. 2018; 495: 2369-75. 